\title{
Synchronization of Switched Interval Networks and Applications to Chaotic Neural Networks
}

\author{
Jinde Cao, ${ }^{1,2}$ Abdulaziz Alofi, ${ }^{2}$ Abdullah Al-Mazrooei, ${ }^{2}$ and Ahmed Elaiw ${ }^{2}$ \\ ${ }^{1}$ Department of Mathematics, Southeast University, Nanjing 210096, China \\ ${ }^{2}$ Department of Mathematics, Faculty of Science, King Abdulaziz University, Jeddah 21589, Saudi Arabia
}

Correspondence should be addressed to Jinde Cao; jdcao@seu.edu.cn

Received 11 November 2013; Accepted 17 November 2013

Academic Editor: Xinsong Yang

Copyright (C) 2013 Jinde Cao et al. This is an open access article distributed under the Creative Commons Attribution License, which permits unrestricted use, distribution, and reproduction in any medium, provided the original work is properly cited.

\begin{abstract}
This paper investigates synchronization problem of switched delay networks with interval parameters uncertainty, based on the theories of the switched systems and drive-response technique, a mathematical model of the switched interval drive-response error system is established. Without constructing Lyapunov-Krasovskii functions, introducing matrix measure method for the first time to switched time-varying delay networks, combining Halanay inequality technique, synchronization criteria are derived for switched interval networks under the arbitrary switching rule, which are easy to verify in practice. Moreover, as an application, the proposed scheme is then applied to chaotic neural networks. Finally, numerical simulations are provided to illustrate the effectiveness of the theoretical results.
\end{abstract}

\section{Introduction}

It is well known that switched system, as a special class of hybrid system [1], is a dynamical system that consists of a finite number of subsystems and a logical rule that orchestrates switching between these subsystems [2-4] has attracted significant attention and successfully been applied to many fields such as artificial intelligence, high speed signal processing, and gene selection in a DNA microarray analysis. Recently, the stability and synchronization problem of switched networks have gained much attention [5-7]. In [6], based on switching analysis techniques and the comparison principle, the exponential synchronization criteria were derived for coupled switched neural networks with modedependent impulsive effects and delay. Authors considered the problems of passivity and pacification for a class of uncertain switched systems with stochastic disturbance and time-varying delay, based on average dwell time approach, free-weighting matrix method, and Jensen's integral inequality, delay-dependent sufficient conditions were obtained to guarantee that the proposed switched systems were robustly mean-square exponentially stable and stochastically passive in terms of linear matrix inequalities [7]. Most of these works results on switched system are based on the Lyapunov theory; however, as we all know, it is difficult to construct a proper common or multiple Lyapunov function for a switched system; hence, in this paper, we will adopt matrix measure theory and Halanay inequality technique instead of constructing Lyapunov function to study the global exponential synchronization of switched networks, what is more, it is easy to verify the proposed conditions.

On the other hand, time delay often exists in nature, which may lead to instability, and it should be considered in mathematical model. Due to unavoidable factors, such as bifurcation and chaos, the networks model certainly involved uncertainties such as perturbations and component variations, which can greatly affect the dynamical behaviors of networks. Robust stability analysis of delayed networks with parameter uncertainties have been widely studied [810]. In [8], authors investigated switched recurrent neural networks (SRNNs) with time-varying norm bounded uncertainties, global asymptotic stability of periodic solution for all admissible parametric uncertainties are derived by taking the relationship between the terms in the Leibniz-Newton formula into account. It should be emphasized that almost all results treated of the robust stability for switched networks with norm-bounded uncertainty in the existing literature. To the best of our knowledge, few researchers deal with 
robust synchronization of switched networks with intervals parameters despite its potential and practical importance. Therefore, it is of great importance to study the global exponential synchronization of switched delay networks with interval parameters uncertainty.

Chaos implies extreme sensitivity to initial conditions; it can be observed in many real-world and has been widely application in secure communication, telecommunications, biological networks, artificial neural networks, and so forth $[11,12]$. To synchronize two chaotic systems were mistakenly considered to be impossible before the pioneer work of Pecora and Carrol [13] on chaos control, and they proposed the drive-response concept to reach the synchronization of coupled chaotic systems. Recently, chaos control and synchronization attract more and more researchers' attentions from various fields [14-18]. So far, kinds of effective approaches and techniques have been proposed for synchronization of chaotic systems including adaptive control [14], feedback control [15], switching control [16], impulsive control $[17,18]$, and others. In [18], global exponential synchronization stability in an array of linearly diffusively coupled reaction-diffusion neural networks with time-varying delays is investigated by adding impulsive controller to a small fraction of nodes, and a new analysis method is developed to overcome the difficulty resulting from the fact that the impulsive controller affects only the dynamical behaviors of the controlled nodes; the proposed results show that designing an appropriate pinning-impulsive controller to realize the synchronization goal as long as a conventional state feedback pinning controller or an adaptive pinning controller can achieve the synchronization goal by controlling the same nodes. In spite of these advances in studying synchronization of chaotic system, synchronization of switched systems with chaotic system as its subsystem under arbitrary switching rule has not been investigated in the literature.

Motivated by the preceding discussion, the aim of this paper is to study the global exponential drive-response synchronization problem for a class of switched interval networks with time-varying delay, instead of LyapunovKrasovskii methods, based on matrix measure theory, Halanay inequality technique, designing the coupling control gain matrix, several synchronization criteria are presented for switched interval networks under the arbitrary switching rule, which are easy to verify in practice. Simulations are given to demonstrate the validity of proposed results. The main contributions of this paper can be highlighted as follows. (1) Consider the interval parameters fluctuation; a new mathematical model of the switched coupled networks with parameters in interval is established, which presents more practical significance of our current research. (2) Introducing matrix measure method and Halanay inequality technique to switched system, without constructing Lyapunov function, the proposed results can easily be verified. (3) The proposed results can be applied to chaotic system; an interesting example shows that the switched networks can reach synchronization even if each subsystem of switched systems is a chaotic system; this further means that our results can generalize the previous results. (4) When $N=1$, the switched interval networks change as interval networks and the synchronization criteria of interval networks can be seen as a by-product.

The rest of this paper is organized as follows. In Section 2, the model description and preliminaries are given. Section 3 treats global exponential synchronization problems for switched interval networks with discrete time-varying delay. In Section 4, exponential synchronization criteria for interval networks are developed. An example is presented to demonstrate that the proposed results can be applied to chaotic system in Section 5. Some conclusions are drawn in Section 6.

Notations. Throughout this paper, for any matrix $A, A>$ $0(A<0)$ means that $A$ is positive definite (negative definite), and $A^{T}$ denotes the transpose of $A . \lambda_{\max }(A)$ and $\lambda_{\min }(A)$ denote the maximum and minimum eigenvalue of $A$, respectively. $I$ is the identity matrix. $P C\left(\left[t_{0}-\tau, t_{0}\right] ; R^{n}\right)$ denote the class of piecewise right continuous function $\eta:\left[t_{0}-\tau, t_{0}\right] \rightarrow$ $R^{n}$ with $p$-norm $\|\eta\|_{p}=\sup _{t_{0}-\tau \leq s \leq t_{0}}\|\eta(s)\|_{p}$. Matrices, if their

dimensions are not explicitly stated, are assumed to have compatible dimensions for algebraic operations.

\section{Model Description and Preliminaries}

Consider a general class of interval networks with timevarying delay described as follows:

$$
\begin{array}{r}
\dot{x}(t)=-A x(t)+B_{1} g_{1}(x(t))+B_{2} g_{2}(x(t-\tau(t)))+J, \\
A \in A_{l}, \quad B_{k} \in B_{l}^{(k)}, \quad k=1,2,
\end{array}
$$

where $x(t)=\left(x_{1}(t), \ldots, x_{n}(t)\right)^{T} \in R^{n}$ is the vector of neuron states; $g_{i}(x)=\left(g_{i 1}\left(x_{1}\right), \ldots, g_{i n}\left(x_{n}\right)\right)^{T}: R^{n} \rightarrow R^{n}, i=$ 1,2 , are the vector-valued neuron activation functions; $\tau(t)$ is the transmission time-varying delay; $J=\left(J_{1}, \ldots, J_{n}\right)^{T}$ is a constant external input vector. $A=\operatorname{diag}\left(a_{1}, \ldots, a_{n}\right)$ is $n \times n$ constant diagonal matrices, $a_{i}>0, i=1, \ldots, n$, are the neural self-inhibitions; $B_{k}=\left(b_{i j}^{(k)}\right) \in R^{n \times n}, k=1,2$, are the connection weight matrices, and $A_{l}=[\underline{A}, \bar{A}]=\{A=$ $\left.\operatorname{diag}\left(a_{i}\right): 0<\underline{a}_{i} \leq a_{i} \leq \bar{a}_{i}, i=1,2, \ldots, n\right\}, B_{l}^{(k)}=$ $\left[\underline{B}_{k}, \bar{B}_{k}\right]=\left\{B_{k}=\left(b_{i j}^{(k)}\right): \underline{b}_{i j}^{(k)} \leq b_{i j}^{(k)} \leq \bar{b}_{i j}^{(k)}, i, j=1,2, \ldots, n\right\}$ with $\underline{A}=\operatorname{diag}\left(\underline{a}_{1}, \underline{a}_{2}, \ldots, \underline{a}_{n}\right), \bar{A}=\operatorname{diag}\left(\bar{a}_{1}, \bar{a}_{2}, \ldots, \bar{a}_{n}\right), \underline{B}_{k}=$ $\left(\underline{b}_{i j}^{(k)}\right)_{n \times n}, \bar{B}_{k}=\left(\bar{b}_{i j}^{(k)}\right)_{n \times n}$.

Throughout this paper, the following assumptions are made on $g_{i}(\cdot), i=1,2$, and $\tau(t)$.

$\left(\mathscr{H}_{1}\right)$ : For any two different $s, t \in R$, there exist constants $l_{i j}>0, i=1,2, j=1,2, \ldots, n$, such that

$\left|g_{i j}(s)-g_{i j}(t)\right| \leq l_{i j}|s-t|, \quad i=1,2, \quad j=1, \ldots, n$.

$\left(\mathscr{H}_{2}\right)$ : For any two different $s, t \in R$, there exist constants $l_{i j}>0, i=1,2, j=1,2, \ldots, n$, such that

$0 \leq \frac{g_{i j}(s)-g_{i j}(t)}{s-t} \leq l_{i j}, \quad i=1,2, j=1, \ldots, n$. 
$\left(\mathscr{H}_{3}\right)$ : Time-varying delay $\tau(t)$ satisfies

$$
0 \leq \tau(t) \leq \tau
$$

where $\tau$ is a positive constant.

Using the coupling feedback control to synchronize system (1), the response (slave) system can be designed as

$$
\begin{aligned}
\dot{y}(t)= & -A y(t)+B_{1} g_{1}(y(t)) \\
& +B_{2} g_{2}(y(t-\tau(t)))+J+U(t), \\
& A \in A_{l}, \quad B_{k} \in B_{l}^{(k)}, \quad k=1,2,
\end{aligned}
$$

where $y(t)=\left(y_{1}(t), \ldots, y_{n}(t)\right)^{T}$ is the neuron state of response system; we choose coupling controller as follows:

$$
U(t)=K(y(t)-x(t))
$$

where the matrix $K \in R^{n \times n}$ is the control gain matrix to be designed.

Let error state be $e(t)=y(t)-x(t)$; then error dynamical system between the states of drive system (1) and response system (5) can be derived:

$$
\begin{array}{r}
\dot{e}(t)=-A e(t)+B_{1} f_{1}(e(t))+B_{2} f_{2}(e(t-\tau(t)))+K e(t), \\
A \in A_{l}, \quad B_{k} \in B_{l}^{(k)}, \quad k=1,2,
\end{array}
$$

where $e(t)=\left(e_{1}(t), \ldots, e_{n}(t)\right)^{T}, f_{1}(e(t))=g_{1}(e(t)+x(t))-$ $g_{1}(x(t)), f_{2}(e(t))=g_{2}(e(t)+x(t))-g_{2}(x(t))$.

Based on some transformations [19], the interval error system (7) can be equivalently written as

$$
\begin{aligned}
\dot{e}(t)= & -\left[A_{0}+E_{A} \Sigma_{A} F_{A}\right] e(t)+\left[B_{10}+E_{1} \Sigma_{1} F_{1}\right] f_{1}(e(t)) \\
& +\left[B_{20}+E_{2} \Sigma_{2} F_{2}\right] f_{2}(x(e(t-\tau(t))))+K e(t),
\end{aligned}
$$

where $\Sigma_{A} \in \Sigma, \Sigma_{k} \in \Sigma, k=1,2$.

Consider the following:

$$
\begin{gathered}
\Sigma=\left\{\operatorname{diag}\left[\delta_{11}, \ldots, \delta_{1 n}, \ldots, \delta_{n 1}, \ldots, \delta_{n n}\right] \in R^{n^{2} \times n^{2}}\right. \\
\left.:\left|\delta_{i j}\right| \leq 1, i, j=1,2, \ldots, n\right\}, \\
A_{0}=\frac{\bar{A}+\underline{A}}{2}, \quad H_{A}=\left[\alpha_{i j}\right]_{n \times n}=\frac{\bar{A}-\underline{A}}{2} \\
B_{k 0}=\frac{\bar{B}_{k}+\underline{B}_{k}}{2}, \quad H_{B}^{(k)}=\left[\beta_{i j}\right]_{n \times n}=\frac{\bar{B}_{k}-\underline{B}_{k}}{2},
\end{gathered}
$$

$E_{A}$

$$
=\left[\sqrt{\alpha_{11}} e_{1}, \ldots, \sqrt{\alpha_{1 n}} e_{1}, \ldots, \sqrt{\alpha_{n 1}} e_{n}, \ldots, \sqrt{\alpha_{n n}} e_{n}\right]_{n \times n^{2}},
$$

$F_{A}$

$$
=\left[\sqrt{\alpha_{11}} e_{1}, \ldots, \sqrt{\alpha_{1 n}} e_{n}, \ldots, \sqrt{\alpha_{n 1}} e_{1}, \ldots, \sqrt{\alpha_{n n}} e_{n}\right]_{n^{2} \times n}^{T},
$$

$E_{k}$

$$
=\left[\sqrt{\beta_{11}^{(k)}} e_{1}, \ldots, \sqrt{\beta_{1 n}^{(k)}} e_{1}, \ldots, \sqrt{\beta_{n 1}^{(k)}} e_{n}, \ldots, \sqrt{\beta_{n n}^{(k)}} e_{n}\right]_{n \times n^{2}},
$$

$F_{k}$

$$
=\left[\sqrt{\beta_{11}^{(k)}} e_{1}, \ldots, \sqrt{\beta_{1 n}^{(k)}} e_{n}, \ldots, \sqrt{\beta_{n 1}^{(k)}} e_{1}, \ldots, \sqrt{\beta_{n n}^{(k)}} e_{n}\right]_{n^{2} \times n}^{T},
$$

where $e_{i} \in R^{n}$ denotes the column vector with $i$ th element to be 1 and others to be 0 .

System (8) has an equivalent form by the following:

$$
\begin{aligned}
\dot{e}(t)=- & A_{0} e(t)+B_{10} f_{1}(e(t))+B_{20} f_{2}(e(t-\tau(t))) \\
& +E \Delta(t)+K e(t),
\end{aligned}
$$

where $E=\left[E_{A}, E_{1}, E_{2}\right]$,

$$
\begin{aligned}
\Delta(t) & =\left[\begin{array}{c}
-\Sigma_{A} F_{A} e(t) \\
\Sigma_{1} F_{1} f_{1}(e(t)) \\
\Sigma_{2} F_{2} f_{2}(e(t-\tau(t)))
\end{array}\right] \\
& =\operatorname{diag}\left\{\Sigma_{A}, \Sigma_{1}, \Sigma_{2}\right\}\left[\begin{array}{c}
-F_{A} e(t) \\
F_{1} f_{1}(e(t)) \\
F_{2} f_{2}(e(t-\tau(t)))
\end{array}\right],
\end{aligned}
$$

and $\Delta(t)$ satisfies the following matrix quadratic inequality:

$$
\begin{aligned}
\Delta^{T}(t) \Delta(t) & \leq\left[\begin{array}{c}
e(t) \\
f_{1}(e(t)) \\
f_{2}(e(t-\tau(t)))
\end{array}\right]^{T}\left[\begin{array}{c}
F_{A}^{T} \\
F_{1}^{T} \\
F_{2}^{T}
\end{array}\right] \\
& \times\left[\begin{array}{c}
F_{A}^{T} \\
F_{1}^{T} \\
F_{2}^{T}
\end{array}\right]^{T}\left[\begin{array}{c}
e(t) \\
f_{1}(e(t)) \\
f_{2}(e(t-\tau(t)))
\end{array}\right] .
\end{aligned}
$$

The switched interval networks with time-varying delay consist of a set of interval networks with discrete timevarying delay and a switching rule [20]. Each of the interval networks is regarded as an individual subsystem. The operation mode of the switched networks is determined by the switching signal. According to (1), the switched interval networks with discrete time-varying delay can be represented as follows:

$$
\begin{gathered}
\dot{x}(t)=-A_{\sigma(t)} x(t)+B_{1_{\sigma(t)}} g_{1}(x(t))+B_{2_{\sigma(t)}} g_{2}(x(t-\tau(t))) \\
+J, \quad A_{\sigma(t)} \in A_{l_{\sigma(t)}}, B_{k_{\sigma(t)}} \in B_{l_{\sigma(t)}}^{(k)}, k=1,2,
\end{gathered}
$$


where $A_{l_{\sigma(t)}}=\left[\underline{A}_{\sigma(t)}, \bar{A}_{\sigma(t)}\right]=\left\{A_{\sigma(t)}=\operatorname{diag}\left(a_{i_{\sigma(t)}}\right): 0<\underline{a}_{i_{\sigma(t)}} \leq\right.$ $\left.a_{i_{\sigma(t)}} \leq \bar{a}_{i_{\sigma(t)}}, i=1,2, \ldots, n\right\}, B_{l_{\sigma(t)}}^{(k)}=\left[\underline{B}_{k_{\sigma(t)}}, \bar{B}_{k_{\sigma(t)}}\right]=\left\{B_{k_{\sigma(t)}}=\right.$ $\left.\left[b_{i j_{\sigma(t)}}^{(k)}\right]: \underline{b}_{i j_{\sigma(t)}}^{(k)} \leq b_{i j_{\sigma(t)}}^{(k)} \leq \bar{b}_{i j_{\sigma(t)}}^{(k)}, i, j=1,2, \ldots, n\right\}$ with $\underline{A}_{\sigma(t)}=$ $\operatorname{diag}\left(\underline{a}_{1_{\sigma(t)}}, \underline{a}_{\sigma_{\sigma(t)}}, \ldots, \underline{a}_{n_{\sigma(t)}}\right), \bar{A}_{\sigma(t)}=\operatorname{diag}\left(\bar{a}_{1_{\sigma(t)}}, \bar{a}_{2_{\sigma(t)}}, \ldots, \bar{a}_{n_{\sigma(t)}}\right)$, $\underline{B}_{k_{\sigma(t)}}=\left[\underline{b}_{i j_{\sigma(t)}}^{(k)}\right]_{n \times n}, \bar{B}_{k_{\sigma(t)}}=\left[\bar{b}_{i j_{\sigma(t)}}^{(k)}\right]_{n \times n}$.

Consider the following:

$$
\begin{aligned}
& A_{0_{\sigma(t)}}=\frac{\bar{A}_{\sigma(t)}+\underline{A}_{\sigma(t)}}{2}, \\
& H_{A_{\sigma(t)}}=\left[\alpha_{i j_{\sigma(t)}}\right]_{n \times n}=\frac{\bar{A}_{\sigma(t)}-\underline{A}_{\sigma(t)}}{2}, \\
& B_{k 0_{\sigma(t)}}=\frac{\bar{B}_{k_{\sigma(t)}}+\underline{B}_{k_{\sigma(t)}}}{2} \\
& H_{B_{\sigma(t)}}^{(k)}=\left[\beta_{i j_{\sigma(t)}}\right]_{n \times n}=\frac{\bar{B}_{k_{\sigma(t)}}-\underline{B}_{k_{\sigma(t)}}}{2}, \\
& E_{A_{\sigma(t)}}=\left[\sqrt{\alpha_{11_{\sigma(t)}}} e_{1}, \ldots, \sqrt{\alpha_{1 n_{\sigma(t)}}} e_{1}, \ldots,\right. \\
& \left.\sqrt{\alpha_{n 1_{\sigma(t)}}} e_{n}, \ldots, \sqrt{\alpha_{n n_{\sigma(t)}}} e_{n}\right]_{n \times n^{2}}, \\
& F_{A_{\sigma(t)}}=\left[\sqrt{\alpha_{11_{\sigma(t)}}} e_{1}, \ldots, \sqrt{\alpha_{1 n_{\sigma(t)}}} e_{n}, \ldots\right. \\
& \left.\sqrt{\alpha_{n 1_{\sigma(t)}}} e_{1}, \ldots, \sqrt{\alpha_{n n_{\sigma(t)}}} e_{n}\right]_{n^{2} \times n}^{T}, \\
& E_{k_{\sigma(t)}}=\left[\sqrt{\beta_{11_{\sigma(t)}^{(k)}}^{(k)}} e_{1}, \ldots, \sqrt{\beta_{1 n_{\sigma(t)}}^{(k)}} e_{1}, \ldots,\right. \\
& \left.\sqrt{\beta_{n 1_{\sigma(t)}^{(k)}}} e_{n}, \ldots, \sqrt{\beta_{n n_{\sigma(t)}^{(k)}}} e_{n}\right]_{n \times n^{2}}, \\
& F_{k_{\sigma(t)}}=\left[\sqrt{\beta_{11_{\sigma(t)}^{(k)}}^{(k)}} e_{1}, \ldots, \sqrt{\beta_{1 n_{\sigma(t)}}^{(k)}} e_{n}, \ldots\right. \text {, } \\
& \left.\sqrt{\beta_{n 1_{\sigma(t)}^{(k)}}^{(k)}} e_{1}, \ldots, \sqrt{\beta_{n n_{\sigma(t)}^{(k)}}} e_{n}\right]_{n^{2} \times n}^{T} .
\end{aligned}
$$

$\sigma(t):[0,+\infty) \rightarrow \Gamma=\{1,2, \ldots, N\}$ is the switching signal, which is a piecewise constant function of time. For any $i \in\{1,2, \ldots, N\}, A_{i}=A_{0_{i}}+E_{A_{i}} \Sigma_{A_{i}} F_{A_{i}}$, $B_{k_{i}}=B_{k 0_{i}}+E_{k_{i}} \Sigma_{k_{i}} F_{k_{i}}$, and $\Sigma_{A_{i}} \in \Sigma, \Sigma_{k_{i}} \in \Sigma, k=$ 1,2 . This means that the matrices $\left(A_{\sigma(t)}, B_{1_{\sigma(t)}}, B_{2_{\sigma(t)}}\right)$ are allowed to take values, at an arbitrary time, in the finite set $\left\{\left(A_{1}, B_{1_{1}}, B_{2_{1}}\right),\left(A_{2}, B_{1_{2}}, B_{2_{2}}\right), \ldots,\left(A_{N}, B_{1_{N}}, B_{2_{N}}\right)\right\}$. In this paper, it is assumed that the switching rule $\sigma$ is not known a priori and its instantaneous value is available in real time.

The initial value associated with the switched interval networks is assumed to be $x(s)=\varphi(s), \varphi(s) \in C\left(\left[t_{0}-\right.\right.$ $\left.\left.\tau, t_{0}\right] ; R^{n}\right)$.
Analogously, slave (response) system [21] of switched interval networks should be defined as

$$
\begin{aligned}
& \dot{y}(t)=-A_{\sigma(t)} y(t)+B_{1_{\sigma(t)}} g_{1}(y(t)) \\
&+B_{2_{\sigma(t)}} g_{2}(y(t-\tau(t)))+J+U(t), \\
& A_{\sigma(t)} \in A_{l_{\sigma(t)}}, \quad B_{k_{\sigma(t)}} \in B_{l_{\sigma(t)}}^{(k)}, \quad k=1,2 .
\end{aligned}
$$

The initial value associated with switched response system is assumed to be $y(s)=\psi(s), \psi(s) \in C\left(\left[t_{0}-\tau, t_{0}\right] ; R^{n}\right)$.

From (10), we have the switched interval drive-response error dynamical system as follows:

$$
\begin{aligned}
\dot{e}(t)=- & A_{0_{\sigma(t)}} e(t)+B_{10_{\sigma(t)}} f_{1}(e(t))+B_{20_{\sigma(t)}} f_{2}(e(t-\tau(t))) \\
& +E_{\sigma(t)} \Delta_{\sigma(t)}(t)+K_{\sigma(t)} e(t),
\end{aligned}
$$

where $E_{\sigma}(t)=\left[E_{A_{\sigma}(t)}, E_{1_{\sigma}(t)}, E_{2_{\sigma}(t)}\right]$ and $\Delta_{\sigma}(t)$ satisfies the following quadratic inequality:

$$
\begin{aligned}
\Delta_{\sigma}^{T}(t) \Delta_{\sigma}(t) & \leq\left[\begin{array}{c}
e(t) \\
f_{1}(e(t)) \\
f_{2}(e(t-\tau(t)))
\end{array}\right]^{T}\left[\begin{array}{c}
F_{A_{\sigma}(t)}^{T} \\
F_{1_{\sigma}(t)}^{T} \\
F_{2_{\sigma}(t)}^{T}
\end{array}\right] \\
& \times\left[\begin{array}{c}
F_{A_{\sigma}(t)}^{T} \\
F_{1_{\sigma}(t)}^{T} \\
F_{2_{\sigma}(t)}^{T}
\end{array}\right]^{T}\left[\begin{array}{c}
e(t) \\
f_{1}(e(t)) \\
f_{2}(e(t-\tau(t)))
\end{array}\right] .
\end{aligned}
$$

Define the indicator function $\xi(t)=$ $\left[\xi_{1}(t), \xi_{2}(t), \ldots, \xi_{N}(t)\right]^{T}$, where

$$
\xi_{i}(t)= \begin{cases}1, & \text { when the switched system is } \\ \text { described by the } i \text { th mode } & \\ A_{0_{i}}, B_{k 0_{0}}, k=1,2, E_{i}, K_{i} \\ 0, & \text { otherwise, }\end{cases}
$$

where $i=1,2, \ldots, N$. Therefore, the switched interval error system (16) can also be represented as

$$
\begin{aligned}
\dot{e}(t)=\sum_{i=1}^{N} \xi_{i}(t)\{ & -A_{0_{i}} e(t)+B_{10_{i}} f_{1}(e(t)) \\
& +B_{20_{i}} f_{2}(e(t-\tau(t))) \\
& \left.+E_{i} \Delta_{i}(t)+K_{i} e(t)\right\},
\end{aligned}
$$

where $\sum_{i=1}^{N} \xi_{i}(t)=1$ is satisfied under any switching rules.

To obtain the main results of this paper, the following definitions and lemmas are introduced.

Definition 1. The switched interval system (13) and the corresponding response system (15) are said to be globally exponentially synchronized if there exist scalars $M>0$ and $\alpha>0$, such that

$$
\|y(t)-x(t)\|_{p} \leq M\|\psi-\varphi\| e^{-\alpha\left(t-t_{0}\right)}, \quad t>t_{0} .
$$


Definition 2 (see [22]). The matrix measure of a real square matrix $A=\left(a_{i j}\right)_{n \times n}$ is denoted as follows:

$$
\mu_{p}(A)=\lim _{\varepsilon \rightarrow 0^{+}} \frac{\left\|I_{n}+\varepsilon A\right\|_{p}-1}{\varepsilon},
$$

where $\|\cdot\|_{p}$ is an induced matrix norm and $p=1,2, \infty$.

Remark 3. The matrix norm $\|A\|_{1}=\max _{j} \sum_{i=1}^{n}\left|a_{i j}\right|,\|A\|_{2}=$ $\sqrt{\lambda_{\max }\left(A^{T} A\right)},\|A\|_{\infty}=\max _{i} \sum_{i=1}^{n}\left|a_{i j}\right|$, the corresponding matrix measure $\mu_{1}(A)=\max _{j}\left\{a_{j j}+\sum_{i=1, i \neq j}^{n}\left|a_{i j}\right|\right\}, \mu_{2}(A)=$ $(1 / 2) \lambda_{\max }\left(A^{T}+A\right), \mu_{\infty}(A)=\max _{i}\left\{a_{i i}+\sum_{j=1, j \neq i}^{n}\left|a_{i j}\right|\right\}$.

Lemma 4 (see [22]). The matrix measure $\mu_{p}(\cdot)$ has the following basic properties:
(i) $-\|A\|_{p} \leq \mu_{p}(A) \leq\|A\|_{p}, \forall A \in R^{n \times n}$;
(ii) $\mu_{p}(\alpha A)=\alpha \mu_{p}(A), \forall \alpha>0, A \in R^{n \times n}$;
(iii) $\mu_{p}(A+B) \leq \mu_{p}(A)+\mu_{p}(B), \forall A, B \in R^{n \times n}$.

Lemma 5 (see [23]). Let $s(t):\left[t_{0}-\tau, \infty\right) \rightarrow[0, \infty)$ be a continuous function, and, for all $t \geq t_{0}$, we have

$$
D^{+} s(t) \leq-a s(t)+b \sup _{t-\tau \leq \theta \leq t} s(\theta) .
$$

If $a>b>0$, then

$$
s(t) \leq \sup _{t_{0}-\tau \leq \theta \leq t_{0}} s(\theta) e^{-\lambda\left(t-t_{0}\right)}, \quad t \geq t_{0},
$$

where $\lambda>0$ is the unique positive solution of the equation $\lambda-$ $a+b e^{\lambda \tau}=0$.

Lemma 6 (see [24]). Under assumption $\left(\mathscr{H}_{2}\right)$, for any matrix $A \in R^{n \times n}$ we have the following inequality:

$$
\mu_{p}(A F(e(t))) \leq \mu_{p}\left(A^{*} L\right)
$$

where $F(e(t))=\operatorname{diag}\left(f_{11}\left(e_{1}(t)\right) / e_{1}(t), \ldots, f_{1 n}\left(e_{n}(t)\right) / e_{n}(t)\right)$, $L=\operatorname{diag}\left(l_{11}, l_{12}, \ldots, l_{1 n}\right), p=1, \infty$,

$$
A^{*}=\left(a_{i j}^{*}\right)_{n \times n} \begin{cases}\max \left(0, a_{i i}\right), & i=j \\ a_{i j}, & i \neq j .\end{cases}
$$

\section{Synchronization Criteria for Switched Interval Networks}

In this section, we will consider the global exponential synchronization of switched interval networks (13), without constructing Lyapunov-Krasovskii functional, by using matrix measure and Halanay inequality, designing suitable control gain matrix $K_{i}$, global exponential stability criteria are derived for switched interval drive-response error system (16) under any arbitrary switched rule; that is to say, the switched interval networks (13) synchronize with the response system (15).
Theorem 7. Under the assumptions $\left(\mathscr{H}_{1}\right)$ and $\left(\mathscr{H}_{3}\right)$, the switched interval networks (13) will globally exponentially synchronize with the response system (15) under arbitrary switched rule, if control gain matrix $K_{i}$ satisfies

$$
\begin{aligned}
& -\mu_{p}\left(-A_{0_{i}}+K_{i}\right)-l\left\|B_{10_{i}}\right\|_{p}-l\left\|E_{i}\right\|_{p}\left\|F_{A_{i}}\right\|_{p}-l\left\|E_{i}\right\|_{p}\left\|F_{1_{i}}\right\|_{p} \\
& \geq l\left\|B_{20_{i}}\right\|_{p}+l\left\|E_{i}\right\|_{p}\left\|F_{2_{i}}\right\|_{p}>0,
\end{aligned}
$$

where $i=1,2, \ldots, N, p=1,2, \infty, l=\max _{1 \leq j \leq n}\left\{l_{k j}\right\}, k=1,2$.

Proof. Calculating the time derivative of $\|e(t)\|_{p}$ along the solution of the system (19), it can follow that

$$
\begin{aligned}
& \lim _{h \rightarrow 0^{+}} \frac{\|e(t+h)\|_{p}-\|e(t)\|_{p}}{h} \\
& =\lim _{h \rightarrow 0^{+}} \frac{\|e(t)+h \dot{e}(t)+o(h)\|_{p}-\|e(t)\|_{p}}{h} \\
& =\lim _{h \rightarrow 0^{+}} \frac{1}{h}\{\| e(t) \\
& +h\left(\sum _ { i = 1 } ^ { N } \xi _ { i } ( t ) \left[-A_{0_{i}} e(t)+B_{10_{i}} f_{1}(e(t))\right.\right. \\
& +B_{20_{i}} f_{2}(e(t-\tau(t))) \\
& \left.\left.+E_{i} \Delta_{i}(t)+K_{i} e(t)\right]\right) \\
& \left.+o(h)\left\|_{p}-\right\| e(t) \|_{p}\right\} \\
& \leq \lim _{h \rightarrow 0^{+}} \sum_{i=1}^{N} \xi_{i}(t)\left\{\frac{\left\|I+h\left(-A_{0_{i}}+K_{i}\right)\right\|_{p}-1}{h}\right. \\
& \times\|e(t)\|_{p}+\left\|B_{10_{i}}\right\|_{p} \\
& \times\left\|f_{1}(e(t))\right\|_{p}+\left\|B_{20_{i}}\right\|_{p} \\
& \times\left\|f_{2}(e(t-\tau(t)))\right\|_{p} \\
& \left.+\left\|E_{i}\right\|_{p}\left\|\Delta_{i}(t)\right\|_{p}\right\} \text {. }
\end{aligned}
$$

Using assumption $\left(\mathscr{H}_{1}\right)$, we yield

$$
\begin{aligned}
&\left\|f_{1}(e(t))\right\|_{p} \leq l\|e(t)\|_{p} \\
&\left\|f_{2}(e(t-\tau(t)))\right\|_{p} \leq l\|e(t-\tau(t))\|_{p} \\
&\left\|\Delta_{i}(t)\right\|_{p} \leq\left\|F_{A_{i}}\right\|_{p}\|e(t)\|_{p}+\left\|F_{1_{i}}\right\|_{p}\left\|f_{1}(e(t))\right\|_{p} \\
&+\left\|F_{2_{i}}\right\|_{p}\left\|f_{2}(e(t-\tau(t)))\right\|_{p}
\end{aligned}
$$




$$
\begin{aligned}
\leq & \left\|F_{A_{i}}\right\|_{p}\|e(t)\|_{p}+l\left\|F_{1_{i}}\right\|_{p}\|e(t)\|_{p} \\
& +l\left\|F_{2_{i}}\right\|_{p}\|e(t-\tau(t))\|_{p} .
\end{aligned}
$$

In the light of (27)-(28), for any $i=1,2, \ldots, N$, we obtain

$$
\begin{aligned}
& \lim _{h \rightarrow 0^{+}} \frac{\|e(t+h)\|_{p}-\|e(t)\|_{p}}{h} \\
& \leq \lim _{h \rightarrow 0^{+}} \sum_{i=1}^{N} \xi_{i}(t)\left\{\frac{\left\|I+h\left(-A_{0_{i}}+K_{i}\right)\right\|_{p}-1}{h}\|e(t)\|_{p}\right. \\
& +\left\|B_{10_{i}}\right\|_{p}\left\|f_{1}(e(t))\right\|_{p} \\
& +\left\|B_{20_{i}}\right\|_{p}\left\|f_{2}(e(t-\tau(t)))\right\|_{p} \\
& \left.+\left\|E_{i}\right\|_{p}\left\|\Delta_{i}(t)\right\|_{p}\right\} \\
& \leq \sum_{i=1}^{N} \xi_{i}(t)\left\{\mu_{p}\left(-A_{0_{i}}+K_{i}\right)\|e(t)\|_{p}\right. \\
& +l\left\|B_{10_{i}}\right\|_{p}\|e(t)\|_{p} \\
& +l\left\|B_{20_{i}}\right\|_{p}\|e(t-\tau(t))\|_{p} \\
& +\left\|E_{i}\right\|_{p}\left\|F_{A_{i}}\right\|_{p}\|e(t)\|_{p} \\
& +l\left\|E_{i}\right\|_{p}\left\|F_{1_{i}}\right\|_{p}\|e(t)\|_{p} \\
& \left.+l\left\|E_{i}\right\|_{p}\left\|F_{2_{i}}\right\|_{p}\|e(t-\tau(t))\|_{p}\right\} \\
& \leq \sum_{i=1}^{N} \xi_{i}(t)\left\{\left(\mu_{p}\left(-A_{0_{i}}+K_{i}\right)+l\left\|B_{10_{i}}\right\|_{p}\right.\right. \\
& \left.+\left\|E_{i}\right\|_{p}\left\|F_{A_{i}}\right\|_{p}+l\left\|E_{i}\right\|_{p}\left\|F_{1_{i}}\right\|_{p}\right) \\
& \times\|e(t)\|_{p}+\left(l\left\|B_{20_{i}}\right\|_{p}+l\left\|E_{i}\right\|_{p}\left\|F_{2_{i}}\right\|_{p}\right) \\
& \left.\times\|e(t-\tau(t))\|_{p}\right\} \\
& \leq\left(\mu_{p}\left(-A_{0_{i}}+K_{i}\right)+l\left\|B_{10_{i}}\right\|_{p}\right. \\
& \left.+\left\|E_{i}\right\|_{p}\left\|F_{A_{i}}\right\|_{p}+l\left\|E_{i}\right\|_{p}\left\|F_{1_{i}}\right\|_{p}\right)\|e(t)\|_{p} \\
& +\left(l\left\|B_{20_{i}}\right\|_{p}+l\left\|E_{i}\right\|_{p}\left\|F_{2_{i}}\right\|_{p}\right)\|e(t-\tau(t))\|_{p} .
\end{aligned}
$$

According to definition of upper-right Dini derivative, we have

$$
\begin{aligned}
D^{+}\|e(t)\|_{p} \leq & \left(\mu_{p}\left(-A_{0_{i}}+K_{i}\right)+l\left\|B_{10_{i}}\right\|_{p}\right. \\
& \left.+\left\|E_{i}\right\|_{p}\left\|F_{A_{i}}\right\|_{p}+l\left\|E_{i}\right\|_{p}\left\|F_{1_{i}}\right\|_{p}\right) \\
& \times\|e(t)\|_{p}+\left(l\left\|B_{20_{i}}\right\|_{p}+l\left\|E_{i}\right\|_{p}\left\|F_{2_{i}}\right\|_{p}\right) \\
& \times \sup _{t-\tau \leq s \leq t}\|e(s)\|_{p} .
\end{aligned}
$$

Let $a=-\mu_{p}\left(-A_{0_{i}}+K_{i}\right)-l\left\|B_{10_{i}}\right\|_{p}-\left\|E_{i}\right\|_{p}\left\|F_{A_{i}}\right\|_{p}-l\left\|E_{i}\right\|_{p}\left\|F_{1_{i}}\right\|_{p}$ and $b=l\left\|B_{20_{i}}\right\|_{p}+l\left\|E_{i}\right\|_{p}\left\|F_{2_{i}}\right\|_{p}$, from condition (26) and Lemma 5, one can obtain

$$
\|e(t)\|_{p} \leq \sup _{t_{0}-\tau \leq s \leq t_{0}}\|e(s)\|_{p} e^{-r\left(t-t_{0}\right)}, \quad t \geq t_{0}
$$

where $r>0$ is the unique positive solution of the equation $r-a+b e^{r \tau}=0$.

Therefore, $e(t)$ converges exponentially to zero with a convergence rate of $r$, and the formula (31) is equivalent to $\|y(t)-x(t)\|_{p} \leq \sup _{t_{0}-\tau \leq s \leq t_{0}}\|\psi-\varphi\|_{p} e^{-r\left(t-t_{0}\right)}$, from Definition 1; this completes the proof of the theorem.

Theorem 8. Under assumptions $\left(\mathscr{H}_{2}\right)$ and $\left(\mathscr{H}_{3}\right)$, global synchronization of the switched interval networks (13) can be achieved under arbitrary switched rule, if designing suitable control gain matrix $K_{i}$ satisfies

$$
\begin{aligned}
& -\mu_{p}\left(-A_{0_{i}}+K_{i}\right)-\mu_{p}\left(B_{10_{i}}^{*} L\right)-l\left\|E_{i}\right\|_{p}\left\|F_{A_{i}}\right\|_{p}-l\left\|E_{i}\right\|_{p}\left\|F_{1_{i}}\right\|_{p} \\
& \geq l\left\|B_{20_{i}}\right\|_{p}+l\left\|E_{i}\right\|_{p}\left\|F_{2_{i}}\right\|_{p}>0
\end{aligned}
$$

where $i=1,2, \ldots, N, p=1, \infty, l=\max _{1 \leq j \leq n}\left\{l_{k j}\right\}(k=1,2)$, and $L=\operatorname{diag}\left(l_{11}, l_{12}, \ldots, l_{1 n}\right)$,

$$
B_{10_{i}}^{*}=\left(b_{m n_{i}}^{(10)^{*}}\right)_{n \times n} \begin{cases}\max \left(0, b_{m n_{i}}^{(10)}\right), & m=n, \\ b_{m n_{i}}^{(10)}, & m \neq n .\end{cases}
$$

Proof. Differentiating $\|e(t)\|_{p}$ with respect to time along the solution of (19), one has

$$
\begin{aligned}
\lim _{h \rightarrow 0^{+}} \frac{\|e(t+h)\|_{p}-\|e(t)\|_{p}}{h} \\
=\lim _{h \rightarrow 0^{+}} \frac{\|e(t)+h \dot{e}(t)+o(h)\|_{p}-\|e(t)\|_{p}}{h}
\end{aligned}
$$




$$
\begin{aligned}
& =\lim _{h \rightarrow 0^{+}} \frac{1}{h}\{\| e(t) \\
& +h\left(\sum _ { i = 1 } ^ { N } \xi _ { i } ( t ) \left[-A_{0_{i}} e(t)+B_{10_{i}} f_{1}(e(t))\right.\right. \\
& +B_{20_{i}} f_{2}(e(t-\tau(t))) \\
& \left.\left.+E_{i} \Delta_{i}(t)+K_{i} e(t)\right]\right)+o(h) \|_{p} \\
& \left.-\|e(t)\|_{p}\right\} \\
& \leq \lim _{h \rightarrow 0^{+}} \sum_{i=1}^{N} \xi_{i}(t)\left\{\left(\| e(t)+h\left(-A_{0_{i}}+K_{i}\right) e(t)\right.\right. \\
& \left.+h B_{10_{i}} f_{1}(e(t))\left\|_{p}-\right\| e(t) \|_{p}\right) \times(h)^{-1} \\
& +\left\|B_{20_{i}}\right\|_{p}\left\|f_{2}(e(t-\tau(t)))\right\|_{p} \\
& \left.+\left\|E_{i}\right\|_{p}\left\|\Delta_{i}(t)\right\|_{p}\right\} \\
& \leq \mu_{p}\left(-A_{0_{i}}+K_{i}+B_{10_{i}} F(e(t))\right)\|e(t)\|_{p} \\
& +\left\|B_{20_{i}}\right\|_{p}\left\|f_{2}(e(t-\tau(t)))\right\|_{p}+\left\|E_{i}\right\|_{p}\left\|\Delta_{i}(t)\right\|_{p} .
\end{aligned}
$$

According to (28), Lemmas 4 and 6, we have

$$
\begin{aligned}
\lim _{h \rightarrow 0^{+}} & \frac{\|e(t+h)\|_{p}-\|e(t)\|_{p}}{h} \\
\leq & \mu_{p}\left(-A_{0_{i}}+K_{i}+B_{10_{i}} F(e(t))\right)\|e(t)\|_{p} \\
& +\left\|B_{20_{i}}\right\|_{p}\left\|f_{2}(e(t-\tau(t)))\right\|_{p}+\left\|E_{i}\right\|_{p}\left\|\Delta_{i}(t)\right\|_{p} \\
\leq & \left(\mu_{p}\left(-A_{0_{i}}+K_{i}\right)+\mu_{p}\left(B_{10_{i}}^{*} L\right)\right)\|e(t)\|_{p} \\
& +l\left\|B_{20_{i}}\right\|_{p}\|e(t-\tau(t))\|_{p}+\left\|E_{i}\right\|_{p}\left\|F_{A_{i}}\right\|_{p}\|e(t)\|_{p} \\
& +l\left\|E_{i}\right\|_{p}\left\|F_{1_{i}}\right\|_{p}\|e(t)\|_{p}+l\left\|E_{i}\right\|_{p}\left\|F_{2_{i}}\right\|_{p}\|e(t-\tau(t))\|_{p} \\
\leq & \left(\mu_{p}\left(-A_{0_{i}}+K_{i}\right)+\mu_{p}\left(B_{10_{i}}^{*} L\right)\right. \\
& \left.+\left\|E_{i}\right\|_{p}\left\|F_{A_{i}}\right\|_{p}+l\left\|E_{i}\right\|_{p}\left\|F_{1_{i}}\right\|_{p}\right)\|e(t)\|_{p} \\
& +\left(l\left\|B_{20_{i}}\right\|_{p}+l\left\|E_{i}\right\|_{p}\left\|F_{2_{i}}\right\|_{p}\right)\|e(t-\tau(t))\|_{p} ;
\end{aligned}
$$

then we get the upper-right Dini derivative of $\|e(t)\|_{p}$ along the solution of system (19) as follows:

$$
\begin{aligned}
D^{+}\|e(t)\|_{p} & \\
\leq & \left(\mu_{p}\left(-A_{0_{i}}+K_{i}\right)\right. \\
& +\mu_{p}\left(B_{10_{i}}^{*} L\right)+\left\|E_{i}\right\|_{p}\left\|F_{A_{i}}\right\|_{p} \\
& \left.+l\left\|E_{i}\right\|_{p}\left\|F_{1_{i}}\right\|_{p}\right)\|e(t)\|_{p} \\
& +\left(l\left\|B_{20_{i}}\right\|_{p}+l\left\|E_{i}\right\|_{p}\left\|F_{2_{i}}\right\|_{p}\right) \sup _{t-\tau \leq s \leq t}\|e(s)\|_{p} .
\end{aligned}
$$

Let $a=-\mu_{p}\left(-A_{0_{i}}+K_{i}\right)-\mu_{p}\left(B_{10_{i}}^{*} L\right)-\left\|E_{i}\right\|_{p}\left\|F_{A_{i}}\right\|_{p}-$ $l\left\|E_{i}\right\|_{p}\left\|F_{1_{i}}\right\|_{p}$ and $b=l\left\|B_{20_{i}}\right\|_{p}+l\left\|E_{i}\right\|_{p}\left\|F_{2_{i}}\right\|_{p}$, from the condition in Theorem 8 and Lemma 5, we get the following:

$$
\|e(t)\|_{p} \leq \sup _{t_{0}-\tau \leq s \leq t_{0}}\|e(s)\|_{p} e^{-r\left(t-t_{0}\right)}, \quad t \geq t_{0}
$$

where $r>0$ is the unique positive solution of the equation $r=a-b e^{r \tau}$.

Therefore, from Definition 1, switched interval networks (13) globally exponentially synchronize to its respond system (15).

\section{Synchronization Criteria of Interval Networks}

It can be observed that only one subsystem is activated when $N=1$, the switched interval networks (13) and corresponding response system (15) degenerate interval networks (1) and its response system (5), respectively. It should be noted that the global exponential synchronization of interval networks can be a by-product; that is, global exponential synchronization criteria for interval networks (1) can be easily derived from Theorems 7 and 8; then we have the following corollaries.

Corollary 9. Under assumptions $\left(\mathscr{H}_{1}\right)$ and $\left(\mathscr{H}_{2}\right)$, when control gain matrix $K$ satisfies

$$
\begin{aligned}
& -\mu_{p}\left(-A_{0}+K\right)-l\left\|B_{10}\right\|_{p}-l\|E\|_{p}\left\|F_{A}\right\|_{p}-l\|E\|_{p}\left\|F_{1}\right\|_{p} \\
& \quad \geq l\left\|B_{20}\right\|_{p}+l\|E\|_{p}\left\|F_{2}\right\|_{p},
\end{aligned}
$$

where $p=1,2, \infty, l=\max _{1 \leq j \leq n}\left\{l_{i j}\right\}, \quad i=1,2$.

Then the interval networks (1) will globally exponentially synchronize with the response system (5).

Proof. Similar to Theorem 7, it is not difficult to get the proof of Corollary 9. 
Corollary 10. Under assumptions $\left(\mathscr{H}_{2}\right)$ and $\left(\mathscr{H}_{3}\right)$, global synchronization of the interval networks (1) can be achieved, if designing suitable control gain matrix $K$ satisfies

$$
\begin{aligned}
& -\mu_{p}\left(-A_{0}+K\right)-\mu_{p}\left(B_{10}^{*} L\right) \\
& -l\|E\|_{p}\left\|F_{A}\right\|_{p}-l\|E\|_{p}\left\|F_{1}\right\|_{p} \\
& \geq l\left\|B_{20}\right\|_{p}+l\|E\|_{p}\left\|F_{2}\right\|_{p}>0,
\end{aligned}
$$

where $p=1, \infty, l=\max _{1 \leq j \leq n}\left\{l_{k j}\right\}(k=1,2)$, and $L=$ $\operatorname{diag}\left(l_{11}, l_{12}, \ldots, l_{1 n}\right)$,

$$
B_{10}^{*}=\left(b_{m n}^{(10)^{*}}\right)_{n \times n} \begin{cases}\max \left(0, b_{m n}^{(10)}\right), & m=n, \\ b_{m n}^{(10)}, & m \neq n .\end{cases}
$$

Proof. The proof of Corollary 10 can be easily obtained from Theorem 8, omitted here.

Remark 11. Matrix measure method has been introduced to switched system, and it is a very useful tool to deal with the stability and synchronization problems of networks and avoid constructing a proper Lyapunov function, which sometimes cannot be found. Moreover, most of the previous results on stability or synchronization are in form of algebra or norm, which limit the scope of nonnegative constants, however, from the definition of matrix measure, one easily knows that matrix measure can balance the effect of positive values and negative values of the matrix. Therefore, method based on matrix measure can obtain the more general synchronization criteria.

\section{An Illustrative Example}

In this section, an example is presented to prove the effectiveness of the theoretical results obtained in Theorem 7 . In addition, it shows that the obtained synchronization criteria can be applied to chaotic neural networks, and even when each subsystem is chaotic neural networks, the switched networks can be reached to synchronization.

Example 1. Consider the second-order switched interval networks with discrete delay in (13) described by

$$
\begin{aligned}
& \dot{x}_{i}(t)=-a_{i_{\sigma(t)}} x_{i}(t)+\sum_{j=1}^{2} b_{i j_{\sigma(t)}}^{(1)} g_{1 j}\left(x_{j}(t)\right) \\
&+ \sum_{j=1}^{2} b_{i j_{\sigma(t)}^{(2)}} g_{2 j}\left(x_{j}(t-\tau(t)) \quad a_{i_{\sigma(t)}} \in\left[\underline{a}_{i_{\sigma(t)}}, \bar{a}_{i_{\sigma(t)}}\right],\right. \\
& b_{i j_{\sigma(t)}^{(k)}}^{(k)} \in\left[\underline{b}_{i j_{\sigma(t)}}^{(k)}, \bar{b}_{i j_{\sigma(t)}}^{(k)}\right], \quad k=1,2, \\
& x_{i}(t)=\varphi_{i}(t), \quad t \in[-\tau, 0], i, j=1,2 .
\end{aligned}
$$

Consider $\sigma(t):[0,+\infty) \rightarrow \Gamma=\{1,2\}, g_{i}(x)=\tan h(x)$, $i=1,2, \tau(t)=1, J=(0,0)^{T}$. Obviously, the assumptions $\mathscr{H}_{1}$ and $\mathscr{H}_{3}$ are satisfied with $l=1$. The networks system parameters are defined as

$$
\begin{array}{ll}
\underline{A}_{1}=\left(\begin{array}{ll}
1.00 & 0.00 \\
0.00 & 1.00
\end{array}\right), & \bar{A}_{1}=\left(\begin{array}{ll}
1.02 & 0.00 \\
0.00 & 1.02
\end{array}\right), \\
\underline{B}_{11}=\left(\begin{array}{cc}
2.00 & -0.10 \\
-5.00 & 4.50
\end{array}\right), & \bar{B}_{11}=\left(\begin{array}{cc}
2.02 & -0.98 \\
-4.98 & 4.52
\end{array}\right), \\
\underline{B}_{21}=\left(\begin{array}{ll}
-1.50 & -0.10 \\
-0.20 & -4.00
\end{array}\right), & \bar{B}_{21}=\left(\begin{array}{ll}
-1.48 & -0.98 \\
-0.18 & -3.98
\end{array}\right), \\
\underline{A}_{2}=\left(\begin{array}{cc}
1.00 & 0.00 \\
0.00 & 1.00
\end{array}\right), & \bar{A}_{2}=\left(\begin{array}{ll}
1.02 & 0.00 \\
0.00 & 1.02
\end{array}\right), \\
\underline{B}_{12}=\left(\begin{array}{cc}
3.00 & 5.00 \\
0.10 & 2.00
\end{array}\right), & \bar{B}_{12}=\left(\begin{array}{cc}
3.02 & 5.02 \\
0.12 & 2.02
\end{array}\right), \\
\underline{B}_{22}=\left(\begin{array}{cc}
-2.50 & 0.20 \\
0.10 & -1.50
\end{array}\right), & \bar{B}_{22}=\left(\begin{array}{cc}
-2.48 & 0.22 \\
0.12 & -1.48
\end{array}\right) .
\end{array}
$$

In the following, we will design control gain matrices $K_{i}(i=1,2)$ for the switched interval networks in this example, chosen as follows:

$$
K_{1}=\left(\begin{array}{cc}
-12 & 4 \\
4 & -20
\end{array}\right), \quad K_{2}=\left(\begin{array}{cc}
-14 & 6 \\
6 & -26
\end{array}\right)
$$

By calculating, we have $-\mu_{p}\left(-A_{0_{1}}+K_{1}\right)-l\left\|B_{10_{1}}\right\|_{p}-$ $l\left\|E_{1}\right\|_{p}\left\|F_{A_{1}}\right\|_{p}-l\left\|E_{1}\right\|_{p}\left\|F_{1_{1}}\right\|_{p}=4.3132 \geq l\left\|B_{20_{1}}\right\|_{p}+$ $l\left\|E_{1}\right\|_{p}\left\|F_{2_{1}}\right\|_{p}=4.0694,-\mu_{p}\left(-A_{0_{2}}+K_{2}\right)-l\left\|B_{10_{2}}\right\|_{p}-$ $l\left\|E_{2}\right\|_{p}\left\|F_{A_{2}}\right\|_{p}-l\left\|E_{2}\right\|_{p}\left\|F_{1_{2}}\right\|_{p}=6.2811 \geq l\left\|B_{20_{2}}\right\|_{p}+$ $l\left\|E_{2}\right\|_{p}\left\|F_{2_{2}}\right\|_{p}=5.0638$, all the assumptions of Theorem 7 hold; therefore, switched drive system (13) can synchronize exponentially toward with response system (15) under any switching rules.

For numerical simulation, let $A_{1}=\underline{A}_{1}, B_{11}=\underline{B}_{11}, B_{21}=$ $\underline{B}_{21}, A_{2}=\underline{A}_{2}, B_{12}=\underline{B}_{12}$, and $B_{22}=\underline{B}_{22}$. In this case, the two subsystems are all chaotic neural networks [25]; Figure 1 displays two chaotic attractors with the initial conditions $\left(x_{11}(t), x_{12}(t)\right)^{T}=(0.1,0.1)^{T},\left(x_{21}(t), x_{22}(t)\right)^{T}=(2.3,0.3)^{T}$, respectively; Figure 2 shows the state trajectories of each variable of the first subsystem, and Figure 3 depicts the temporal evolution of each variable of the second subsystem.

For making numerical simulation of the switched driveresponse error-state system, Figure 4 depicts the time responses of state variables $e_{1}(t)$ and $e_{2}(t)$ from the 30 random constant initial states in the set $[-2,4] \times[-2,4]$ with step $h=0.01$; it reveals that the trajectory of the switched interval error-state system globally exponentially converges to a unique equilibrium $e^{*}=(0,0)^{T}$. This is in accordance with the conclusion of Theorem 7 . 

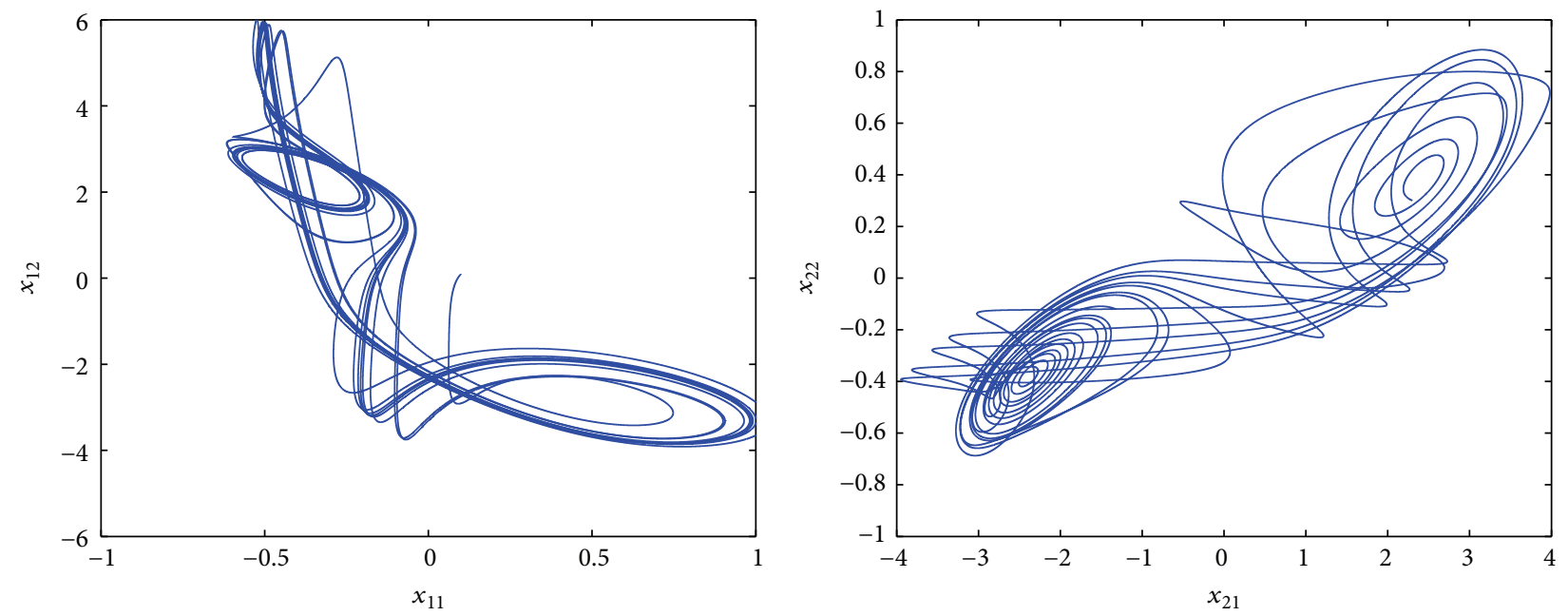

FIGURE 1: Chaotic attractor of two subsystems of switched networks.
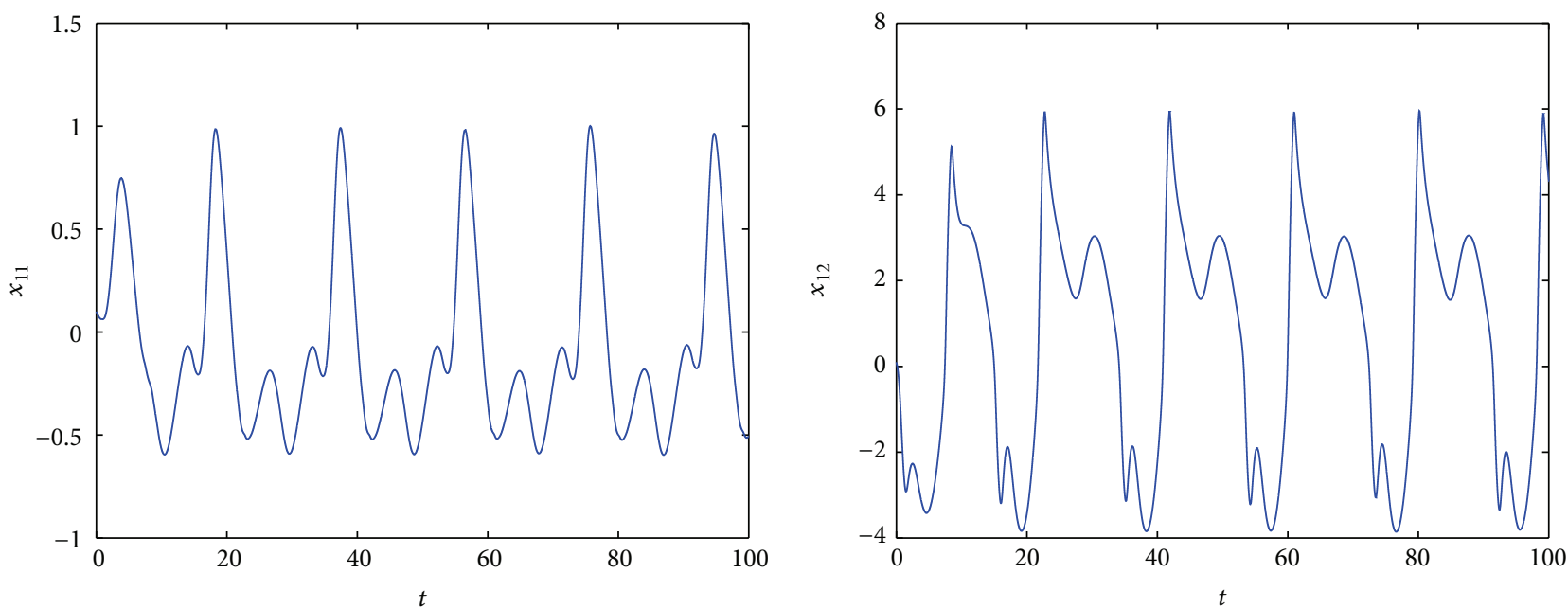

FIGURE 2: The state trajectories $x_{11}$ and $x_{12}$ of the first subsystem.
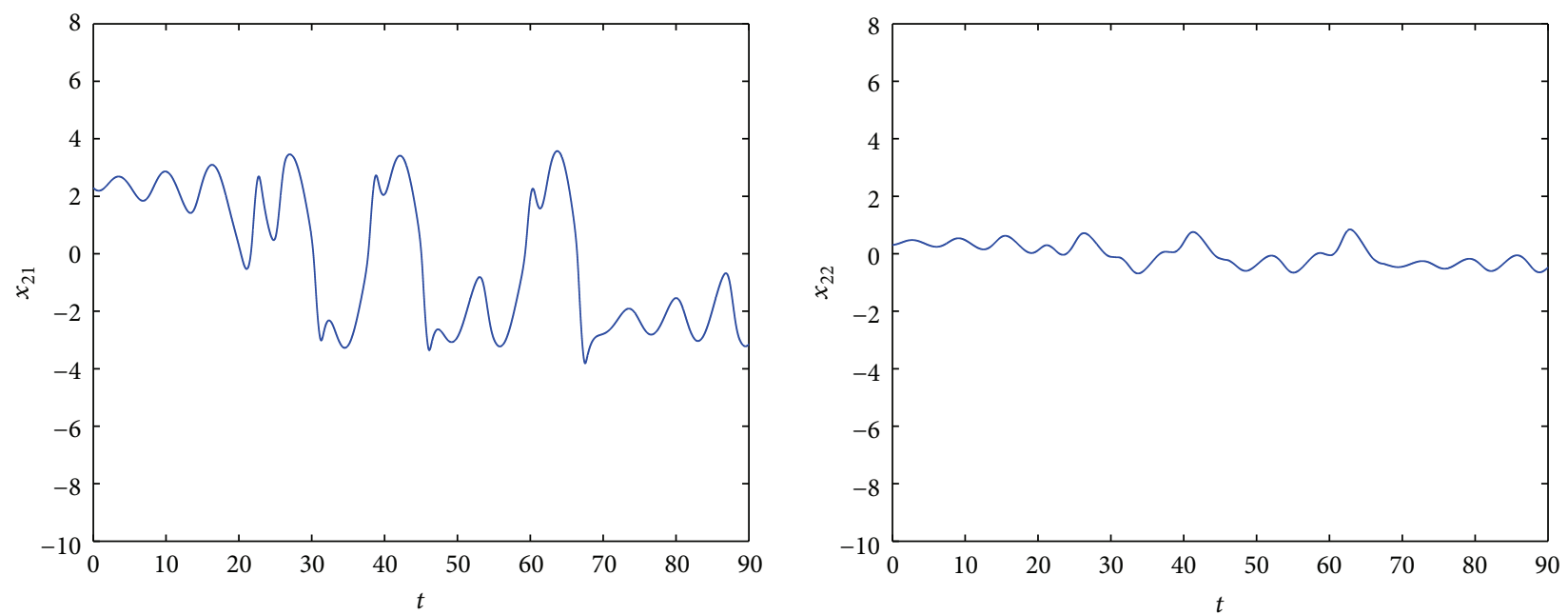

FIGURE 3: The state trajectories $x_{21}$ and $x_{22}$ of the second subsystem. 

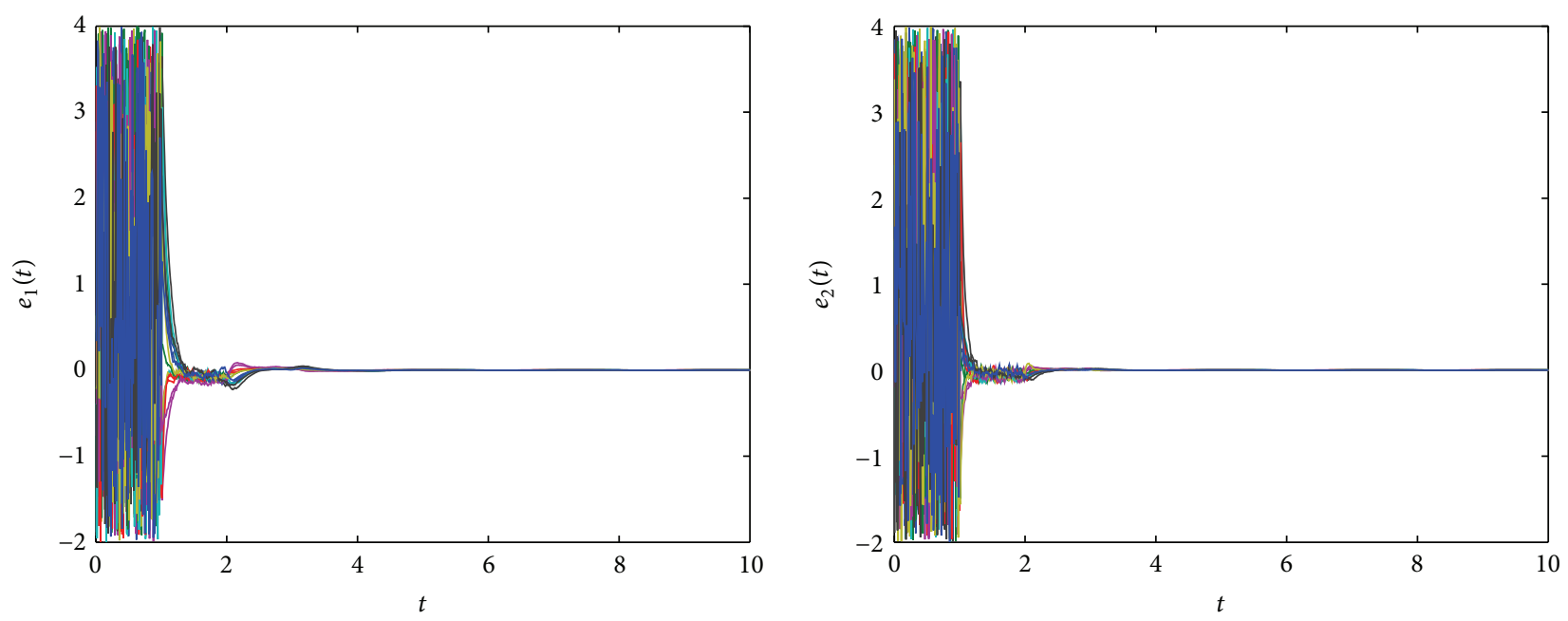

FIGURE 4: Time responses of state variables $e_{1}$ and $e_{2}$ of switched error-state system.

\section{Conclusion}

In this paper, without constructing complex LyapunovKrasovskii functions, we have proposed a new method to study the global exponential synchronization of switched interval delayed networks and designed the coupling control gain matrices $K_{i}$; the derived synchronization criteria for switched interval networks under the arbitrary switching rule are easy to verify in practice. The synchronization criteria for delayed networks with uncertain parameters can be a special case. Additionally, synchronization criteria can be applied to chaotic systems. An interesting example has shown that switched drive systems can synchronize with its response systems even if each subsystem is chaotic neural networks; this further shows that our results improve and extend the existing works. In the near future, we will discuss quasi-synchronization control, dissipativity, and finite-time stochastic stabilization of the switched interval delayed networks [26, 27].

\section{Acknowledgments}

This work was funded by the Deanship of Scientific Research (DSR), King Abdulaziz University (KAU), under Grant 3130/1434/HiCi. The authors, therefore, acknowledge the technical and financial support of KAU.

\section{References}

[1] P. Antsaklis, W. Kohn, A. Nerode, and S. Sastry, Hybrid Systems II, vol. 999 of Lecture Notes in Computer Science, Springer, Berlin, Germany, 1995.

[2] T. X. Brown, "Neural networks for switching," IEEE Communications Magazine, vol. 27, no. 11, pp. 72-81, 1989.

[3] Y. Tsividis, "Switched neural networks," United States Patent no. 4873661, 1989.

[4] D. Liberzon, Switching in Systems and Control, Birkhäauser, Boston, Mass, USA, 2003.
[5] H. Lin and P. J. Antsaklis, "Stability and stabilizability of switched linear systems: a survey of recent results," IEEE Transactions on Automatic Control, vol. 54, no. 2, pp. 308-322, 2009.

[6] W. Zhang, Y. Tang, Q. Miao, and D. Wei, "Exponential synchronization of coupled switched neural networks with modedependent impulsive effects," IEEE Transactions on Neural Networks and Learning Systems, vol. 24, no. 8, pp. 1316-1326, 2013.

[7] J. Lian, P. Shi, and Z. Feng, "Passivity and passification for a class of uncertain switched stochastic time-delay systems," IEEE Transactions on Cybernetics, vol. 43, no. 1, pp. 3-13, 2013.

[8] X. Lou and B. Cui, "Delay-dependent criteria for global robust periodicity of uncertain switched recurrent neural networks with time-varying delay," IEEE Transactions on Neural Networks, vol. 19, no. 4, pp. 549-557, 2008.

[9] P. Balasubramaniam, V. Vembarasan, and R. Rakkiyappan, "Global robust asymptotic stability analysis of uncertain switched Hopfield neural networks with time delay in the leakage term," Neural Computing and Applications, vol. 21, no. 7, pp. 1593-1616, 2012.

[10] O. Faydasicok and S. Arik, "Equilibrium and stability analysis of delayed neural networks under parameter uncertainties," Applied Mathematics and Computation, vol. 218, no. 12, pp. 6716-6726, 2012.

[11] J. F. Heagy, T. L. Carroll, and L. M. Pecora, "Synchronous chaos in coupled oscillator systems," Physical Review E, vol. 50, no. 3, pp. 1874-1885, 1994.

[12] Ö. Morgül and M. Feki, "Synchronization of chaotic systems by using occasional coupling," Physical Review E, vol. 55, no. 5, pp. 5004-5010, 1997.

[13] L. M. Pecora and T. L. Carroll, "Synchronization in chaotic systems," Physical Review Letters, vol. 64, no. 8, pp. 821-824, 1990.

[14] J. Lu, X. Wu, X. Han, and J. Lü, "Adaptive feedback synchronization of a unified chaotic system," Physics Letters A, vol. 329, no. 4-5, pp. 327-333, 2004.

[15] M. T. Yassen, "Controlling chaos and synchronization for new chaotic system using linear feedback control," Chaos, Solitons and Fractals, vol. 26, no. 3, pp. 913-920, 2005. 
[16] S. Cho, M. Jin, T. Y. Kuc, and J. S. Lee, "Control and synchronization of chaos systems using time-delay estimation and supervising switching control," Nonlinear Dynamics, 2013.

[17] J. Yu, C. Hu, H. Jiang, and Z. Teng, "Stabilization of nonlinear systems with time-varying delays via impulsive control," Neurocomputing, vol. 125, pp. 68-71, 2014.

[18] X. Yang, J. Cao, and Z. Yang, "Synchronization of coupled reaction-diffusion neural networks with time-varying delays via pinning-impulsive controller," SIAM Journal on Control and Optimization, vol. 51, no. 5, pp. 3486-3510, 2013.

[19] L. Liu, Z. Han, and W. Li, "Global stability analysis of interval neural networks with discrete and distributed delays of neutral type," Expert Systems with Applications, Part II, vol. 36, no. 3, pp. 7328-7331, 2009.

[20] J. Lian and K. Zhang, "Exponential stability for switched CohenGrossberg neural networks with average dwell time," Nonlinear Dynamics, vol. 63, no. 3, pp. 331-343, 2011.

[21] J. A. K. Suykens, P. F. Curran, and L. O. Chua, "Robust synthesis for master-slave synchronization of Lur'e systems," IEEE Transactions on Circuits and Systems I, vol. 46, no. 7, pp. 841-850, 1999.

[22] M. Vidyasagar, Nonlinear System Analysis, Prentice Hall, Englewood Cliffs, NJ, USA, 1993.

[23] A. Halanay, Differential Equations: Stability, Oscillations, Time Lags, Academic Press, New York, NY, USA, 1966.

[24] W. He and J. Cao, "Exponential synchronization of chaotic neural networks: a matrix measure approach," Nonlinear Dynamics, vol. 55, no. 1-2, pp. 55-65, 2009.

[25] H. Lu, "Chaotic attractors in delayed neural networks," Physics Letters A, vol. 298, no. 2-3, pp. 109-116, 2002.

[26] X. Liu, J. Cao, and W. Yu, "Filippov systems and quasisynchronization control for switched networks," Chaos, vol. 22, no. 3, article 033110, 2012.

[27] X. Liu, N. Jiang, J. Cao, S. Wang, and Z. Wang, "Finitetime stochastic stabilization for BAM neural networks with uncertainties," Journal of the Franklin Institute, vol. 350, no. 8, pp. 2109-2123, 2013. 


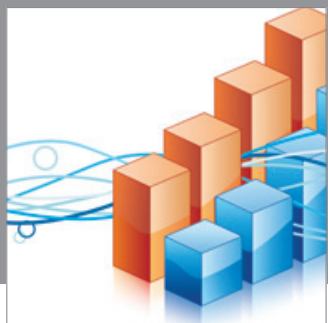

Advances in

Operations Research

mansans

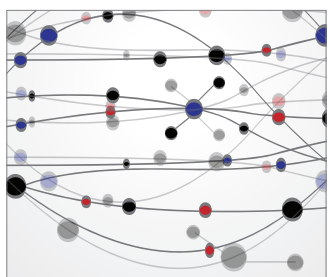

The Scientific World Journal
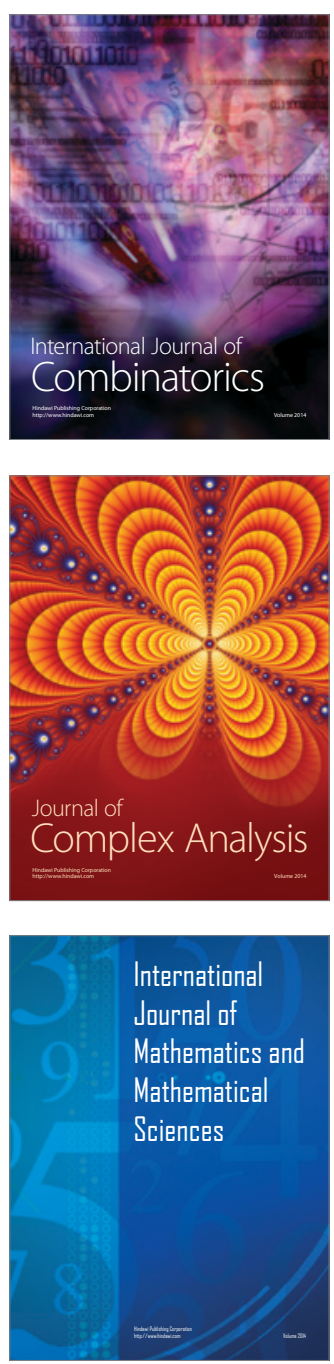
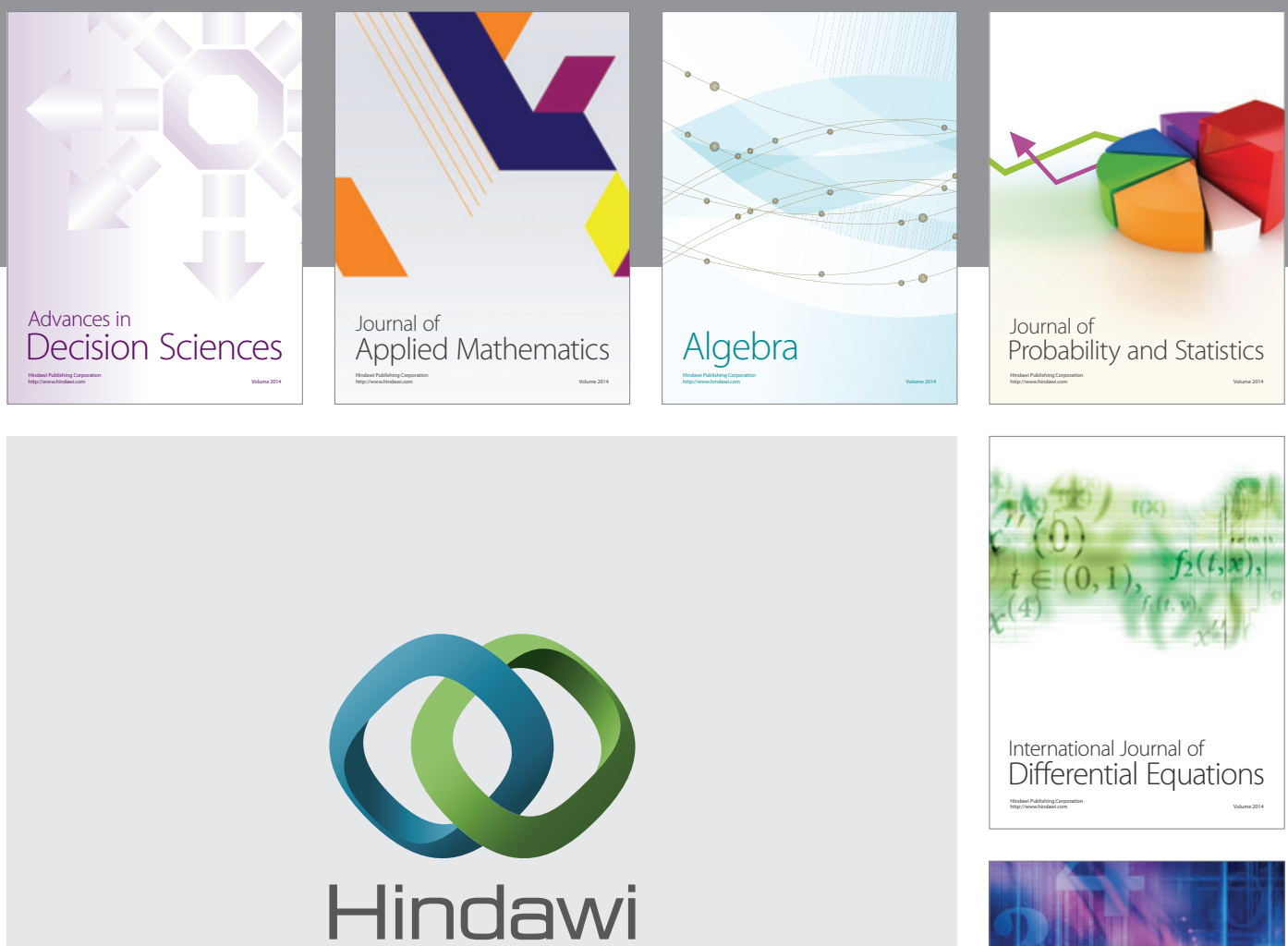

Submit your manuscripts at http://www.hindawi.com
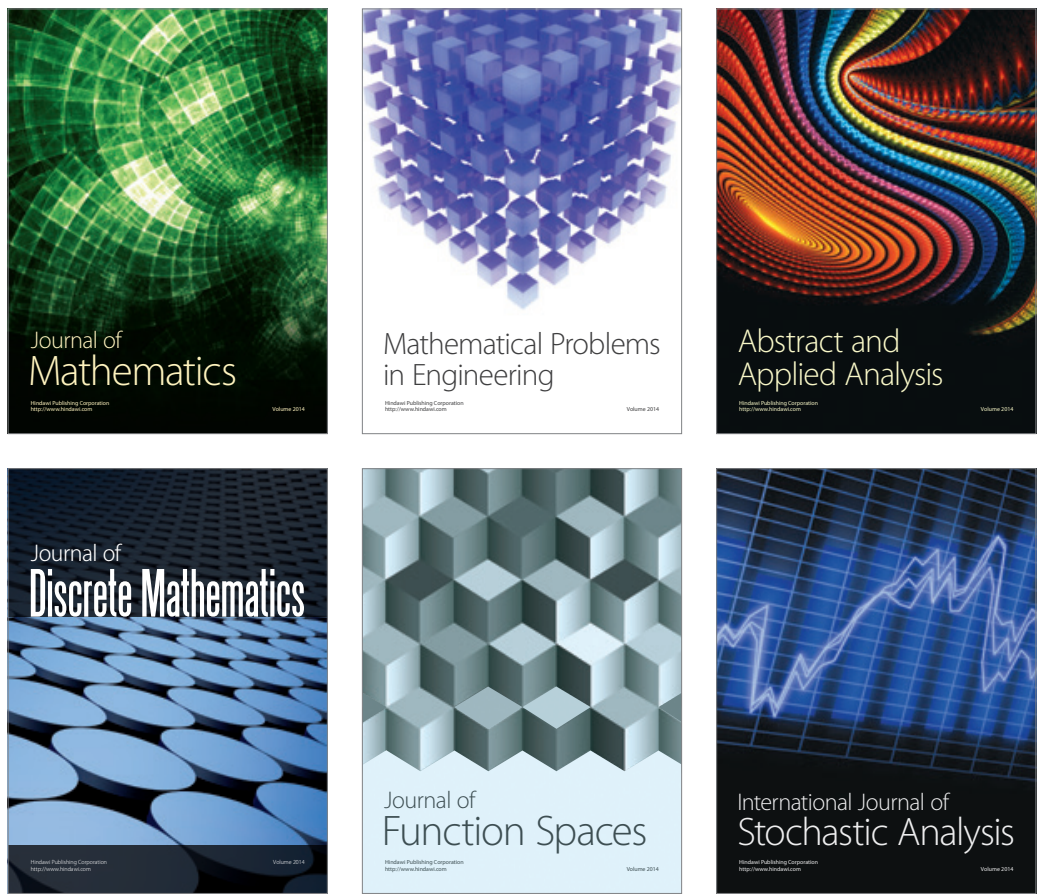

Journal of

Function Spaces

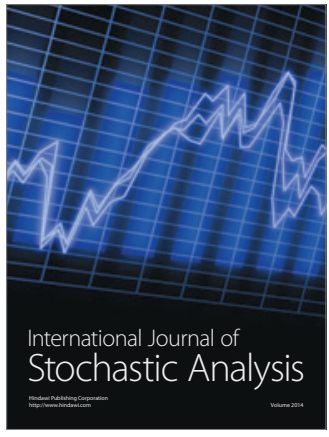

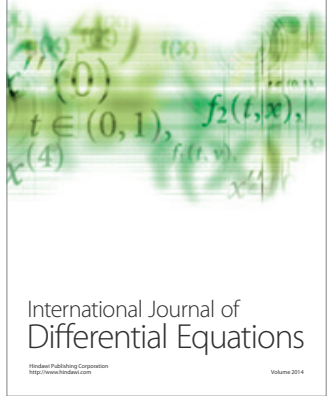
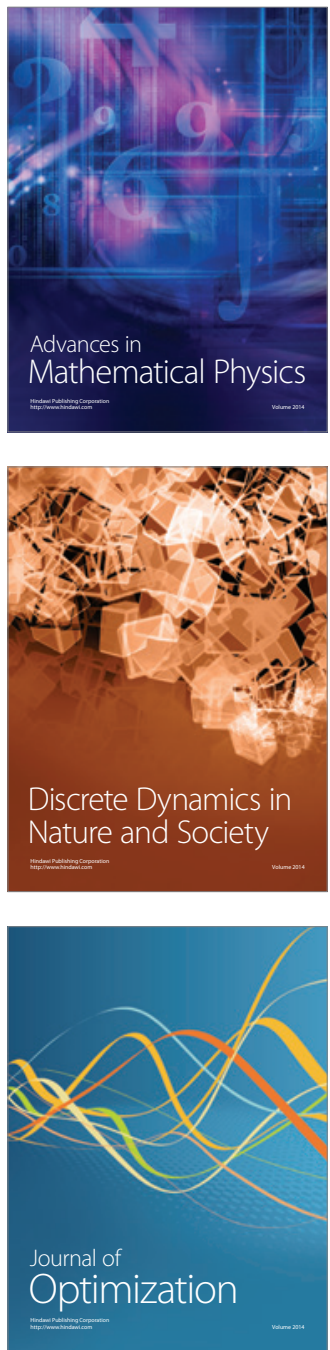